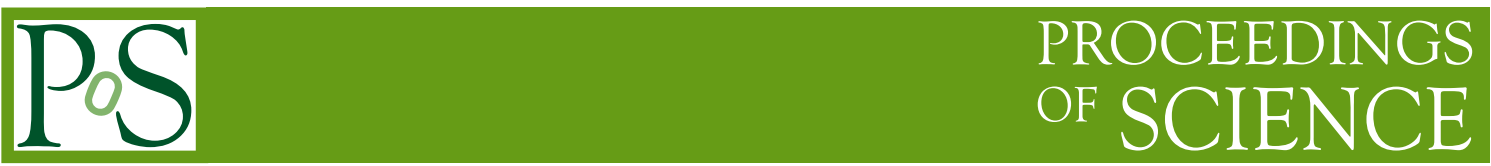

\title{
The KM3NeT Multi-PMT Digital Optical Module
}

\section{R. Bruijn ${ }^{* a b}$ and D. van Eijk ${ }^{a}$ for the KM3NeT Collaboration}

a Nikhef, Science Park, Amsterdam, The Netherlands

b Universiteit van Amsterdam, Instituut voor Hoge-Energie Fysica, Science Park 105, 1098 XG

Amsterdam, The Netherlands

E-mail: rbruijnenikhef.nl, dveijkenikhef.nl

The KM3NeT collaboration is currently constructing the first phase of a cubic kilometer-scale neutrino detector in the Mediterranean Sea. The basic detection element, the Digital Optical Module (DOM), houses 31 three-inch photomultiplier tubes (PMTs) inside a $432 \mathrm{~mm}$ glass sphere. This multi-PMT concept increases the photocathode area by a factor of three compared to a design with a single 10 inch PMT, leading to a significant cost reduction. Moreover, this concept allows for an accurate measurement of the light intensity (photon counting) and offers directional information with an almost isotropic field of view. We will discuss these aspects and the enabling technologies, which include 3D-printed PMT support structures, and custom low-powered PMT bases, which provide the HV and digitization of the analog signal. An FPGA-based readout system transfers all sub-nanosecond timestamped photon signals to shore via optical fibres. The DOM design has been validated and its physics potential has been proven in the operation of prototypes deployed in the French and Italian detector sites at $2500 \mathrm{~m}$ and $3500 \mathrm{~m}$ depth respectively.

The 34th International Cosmic Ray Conference,

30 July- 6 August, 2015

The Hague, The Netherlands

${ }^{*}$ Speaker. 


\section{Introduction}

The first phase of the KM3NeT neutrino telescope [1],[2] is currently under construction. The KM3NeT collaboration aims at studying the flux of extra-terrestrial neutrinos and to discover its sources by detecting Cherenkov light emitted by products of neutrino interactions in the deep waters of the Mediterranean sea. In addition, fundamental properties of neutrinos will be studied, in particular the neutrino mass hierarchy. To achieve these science goals, large volumes of sea water will be instrumented with photo-multplier tubes (PMTs). Following the first phase it is foreseen to build a telescope dedicated to the high-energy neutrino sky, ARCA (Astroparticle Research with cosmics in the Abyss) and a denser detector, ORCA (Oscillation Research with Cosmics in the Abyss), dedicated to a lower neutrino energy range for the study of neutrino properties.

Essential to these efforts is to detect the sparse photons resulting from neutrino interactions with nanosecond timing accuracy and to distinguish them from light emitted by ambient sources, such as potassium decay and bioluminescence, and muons from cosmic ray interactions. In order to protect the PMTs from the hostile environment (pressure, salt water), they are housed in a pressureresistant sphere which contains the power, control, positioning and read-out systems required to precisely measure the photon arrival times and positions. These units are called Digital Optical Modules, or DOMs. Communication and data transport occurs via optical fibres which connect the DOMs via a sea-floor network to shore. To survey the high-energy neutrino sky, 18 DOMs are placed at 37 meters vertically from each other along one detector line, thus instrumenting about 700 meters. Such a vertical line is referred to as a detection unit (DU). Typically, the DUs are spaced 100 meters apart. In this way, a three-dimensional grid of DOMs is created. The current phase will consist of 24 detection units at the KM3NeT-Italy (about 3500m deep) site and 1 at the KM3NeT-France (about 2400m deep) site. To study neutrino properties, more compact designs are considered with a vertical DOM spacing ranging from 6 to 15 meters and a horizontal DU spacing in the order of 20 meters. For the current phase, 6 of these compact DUs will be installed at the KM3NeT-France site. Subsequent phases (e.g. ARCA/ORCA) will consist of one or more building blocks of 115 DUs. The KM3NeT DOMs allow to cost-effectively create a large effective photocathode area with direction sensitivity and background rejection capabilities already at DOM level.

\section{Design of the KM3NeT DOM}

General constraints on the detector and thus DOM design follow from the scientific goals and environmental conditions. The total photo collection area must be optimized as the expected astrophysical neutrino interaction rate is very low. Increasing the overburden decreases the background from cosmic ray muons, but puts the DOMs in a more hostile environment. Besides the background from cosmic ray muons which is 8 orders of magnitude larger than an expected signal, the environment introduces an overwhelming optical background from bioluminescence and potassium decay. To optimally extract a signal from these backgrounds, nanosecond timing accuracy is required. The DOMs must withstand pressures up to about 500 bar (depending on the site), be resistant to corrosion and to stress (vibration, shocks) during handling and deployment. The lifetime of the detectors should exceed 10 years and since servicing and repairing of DOMs is difficult and expensive, the 
reliability of the DOMs should be high.

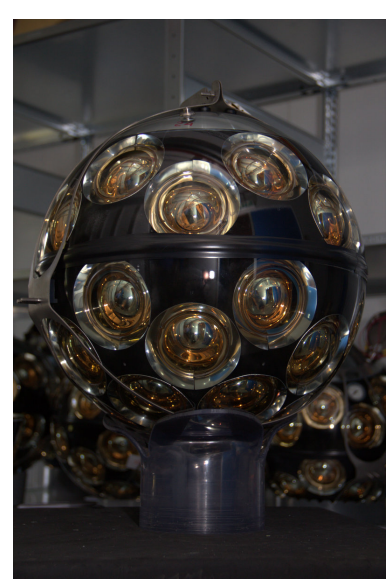

Figure 1: A KM3NeT DOM right after assembly.

The design of the KM3NeT DOM differs from the traditional concept of optical modules in large-volume neutrino telescopes which are equiped with single large PMTs in an effort to maximize photocathode surface. The innovative layout consists of a $432 \mathrm{~mm}$ diameter glass sphere which houses 31 small PMTs together with the power, control and digitization electronics, an acoustic detector and a calibration light source. A total photocathode area (about $1400 \mathrm{~cm}^{2}$ ) equivalent to three 10" PMTs is obtained in this way, while profiting from the better timing and amplitude measurement characteristics of small PMTs. Segmenting the total photocathode area enhances the capability to measure the photon flux originating from a source. To first order, photons can be counted by counting the number of PMTs registering a photon hit. By exploiting the coincidences between different (neighbouring) PMTs, the background from uncorrelated photons can be reduced. An almost isotropical angular coverage is achieved with the PMT orientations ranging from vertically down to upwards at an angle of $58^{\circ}$ from horizontal. Due to this coverage, unscattered light from sources with arbitrary orientation from the DOMs can be measured. A DOM is also equipped with a LED beacon [3] with a wavelength of $470 \mathrm{~nm}$ for calibration purposes and an internal acoustic sensor [4],[5] coupled to the glass to allow for acoustic positioning and other purposes [6]. A photo of a DOM right after assembly can be seen in figure 1 .

\subsection{PMTs}

Besides having good performance characteristics, the dimensions of the PMTs should be such that a sufficiently large amount of photocathode area can be mounted in the pressure-resistant glass sphere, while leaving room for the power and read-out systems. Different types of PMTs with comparable performance and slightly varying dimensions are used in the DOM prototypes (ETEL D783FL [7], Hamamatsu R12199-02 [8]). Typical performances are a transit time spread lower than $5 \mathrm{~ns}$ (FWHM), and quantum efficiencies of $22 \%$ at $470 \mathrm{~nm}$ and $27 \%$ at $404 \mathrm{~nm}$. These characteristics meet the requirements to achieve the desired detector performance. The PMTs used in the current KM3NeT phase are the Hamamatsu R12199-02, which have a diameter of $80 \mathrm{~mm}$. The PMTs are operated at a gain of $3 \times 10^{6}$. Dark count rates as measured in the laboratory at room temperature vary typically between 200 and $1500 \mathrm{~Hz}$ with a threshold of 0.3 photoelectrons. The total countrate in-situ, including background, is expected to typically lie in the range from $5 \mathrm{kHz}$ to $10 \mathrm{kHz}$. The PMTs are equipped with a compact active base [9] which serves to feed the PMTs with high-voltage (of order $1 \mathrm{kV}$ ) and to process the PMT signal. High voltage is generated directly on the base by a Cockroft-Walton circuit which is controlled by a application-specific integrated circuit (ASIC) named CoCo [10]. Pulses from the PMT are translated to a time-stamp and the duration that the signal is above a configurable threshold (equivalent to 0.3 photo-electrons). This is implemented in a second ASIC, called PROMiS (PMT Read Out Mixed Signal) [11]. It has a comparator whose threshold can be varied and produces a low-voltage differential signal (LVDS) 
for the duration that the signal is above threshold. By using the time of threshold crossing and the time-over-threshold (ToT), the information on the photon flux at the PMTs can be encoded compactly which reduces the bandwidth use. The gain adjustment is performed for all PMTs by finding the high-voltage setting which gives a ToT of $26.4 \mathrm{~ns}$ for a single photoelectron [12]. Communication with the base is done via the $\mathrm{I} 2 \mathrm{C}$ protocol implemented in the PROMiS ASIC. This allows the setting of the comparator threshold and the high-voltage. A unique identification number of the base provided by PROMiS can be read-out. All PMTs are calibrated and characterized (HV setting, dark count) before being used in the production of DOMs [12].

\subsection{Mechanical structure}

Most of the components of a DOM are contained within the aforementioned $432 \mathrm{~mm}$ glass sphere (Vitrovex, a low-activity borosilicate glass), as is shown in the exploded view of a DOM (Fig. 2). The glass thickness is $14 \mathrm{~mm}$ to withstand the pressure difference between the deep sea and the air pressure at sea level inside the DOM. The sphere is formed by joining two glass hemispheres. The glass sphere meets requirements on the transparency to photons in the relevant range (400-500 nm) with a transmission of larger than $95 \%$ above $350 \mathrm{~nm}$. The PMTs (Fig. 2, H) are arranged in five rings of six PMTs, at angles of $56^{\circ}, 72^{\circ}, 107^{\circ}, 123^{\circ}$ and $148^{\circ}$ degrees from zenith and one PMT points vertically down $\left(180^{\circ}\right)$. In each ring, the PMTs are spaced at $60^{\circ}$ degrees in azimuth and successive rings are shifted by approximately $30^{\circ}$ in azimuth. This creates a large angular coverage. The space at the top of a DOM is not instrumented with PMTs, allowing the installation of a mechanical support for the electronics (Fig. 2, D,E,F) and a passive cooling system (Fig. 2, C). Thus, the lower hemisphere houses 19 PMTs, while the upper hemisphere contains 12 PMTs. The upper glass hemisphere (Fig. 2, B) contains two drilled holes. One hole is instrumented with a vacuum valve for evacuation of air from the inside of a DOM, while in the other hole a penetrator (Fig. 2, A) is mounted. This custom-designed device features a titanium housing and a feedthrough for power cables and an optical fibre for bi-directional communication. This is a crucial component which must withstand the pressure difference between the inside and outside of the sphere. The 31 PMTs are mounted in a nylon support structure (Fig. 2, J) which is manufactured by $3 \mathrm{D}$ printing with the selective laser sintering method, allowing to incorporate multiple functionalities. The support structure defines the PMT positions and the close distance to the glass. The PMT locations are tapered and a silicone O-ring tightly seals around the PMT just below its head. The support structure also provides mounts for reflector rings (92\% reflectance for $375-500 \mathrm{~nm}$ ) [13] (Fig. 2, G) that are placed at an angle of 45 degrees around the PMTs to increase the photon yield (20-40\%) and directionality. The support structure is segmented into an upper and a lower half. Each of the halves is 3D-printed in two parts, which are joined during assembly. While the printed structures are white, they are painted black to reduce the optical background. In addition, the support structures serve several other purposes: the upper support holds a LED flasher pointing upwards for timing calibration between DOMs (the so-called nanobeacon, Fig. 2, I) and a pressure gauge to monitor the air pressure inside the DOM before deployment (Fig. 2, L). The lower support also has a feedthrough for the acoustic sensor which is attached to the glass sphere (Fig 2, K). Optical contact between the PMTs and the glass is ensured bassembly cay a transparent two component silicone gel (Wacker SilGel 612 A/B) which is administered in the space enclosed by the support structure, the PMTs and glass. The support structure is equipped with funnel holes to which tubes 


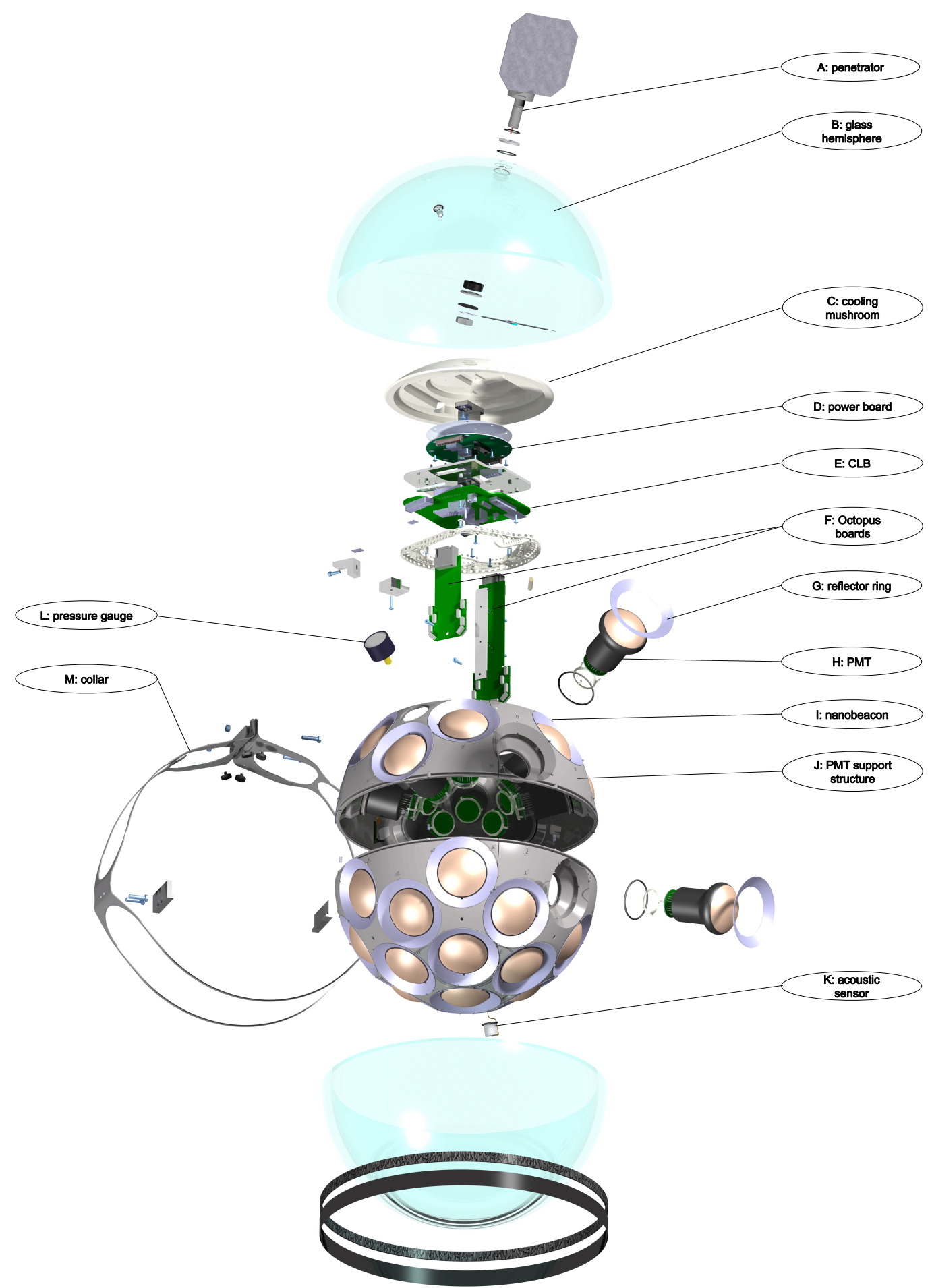

Figure 2: An exploded view of a KM3NeT DOM. The main components are indicated and referenced in the text. 
can be attached in order to facilitate the administering of the gel. Markings printed on the support structure serve to map the PMT positions to specific read-out channels during construction and thus defining the spatial orientation of the PMTs. Additional markings define the relative orientation of the structures and their orientation within the DOM during the assembly process. External to the glass sphere is a titanium collar with associated mechanics (Fig. 2, M), which clamps the sphere and attaches it to two Dyneema ropes that form the structural support of the detector line. The collar also provides a mount for the so-called break-out box (BOB) in which a fibre from the pressure balanced Vertical Electro-Optical Cable (VEOC) running along the detector line is split off. The $\mathrm{BOB}$ also contains a DC/DC converter $(400 \mathrm{~V}$ to $12 \mathrm{~V})$. The power cables and optical fibre enter the DOM via the penetrator. The mechanics of the DU, including the VEOC and the deployment system are described in a different contribution to this conference [19].

\subsubsection{DOM production}

The design features of the DOM have been optimized for functionality and to facilitate assembly. The two halves are constructed separately and are connected as the final construction step when also a slight underpressure is created in the sphere ( 0.2 bar below atmospheric pressure). Splicing of optical fibres is required to create the connection between the laser transceiver and the fibre leading to the outside. DOMs are constructed from components which have been through acceptance tests and are characterized before arrival (e.g. [12]) at the construction location and are thus ready to be used. At specificied moments during the construction, tests are performed; the mounted penetrator is subject to a helium leak test, the fibre splice and resulting optical power are measured and a functional test of all systems is performed before applying the optical gel. After closing, an extensive final acceptance test is performed in a light-tight dark box. The time required to assemble a DOM is dominated by the setting times of the glue (piezo to glass), silicone mastic (support structures, support structure to shell, nanobeacon) and silicone gel (between glass and inner components) and by a waiting period (in darkness) before the dark box test.

All components are identified by either a unique identifier or a batch identifier when appropriate and are registered in a database before arrival at the construction site. A QR code is associated with each unique identifier, which allows for the quick and accurate identification of a component by means of a (handheld) scanner. The construction process is guided by a system which tracks the components integrated in a DOM and enters the information into the database and is cross-checked at the various tests by reading out electronic identifiers and analysing the test data.

\subsection{Read-Out}

Central to the design of the data-acquisition [14] system of the KM3NeT telescope is the alldata-to-shore concept. All digitized photon arrival times $\left(t_{0}\right)$ and ToTs that are recorded by the PMTs are transported via an optical fibre network to shore where a farm of processors applies trigger and selection algorithms to reduce the data. This method allows for sophisticated algorithms to filter the overwhelming background by searching for space-time correlated photon hits which are signatures of neutrino interactions. The expected average photon-counting rate of 5-10 $\mathrm{kHz}$ per PMT will lead to an outgoing data-rate of a few MB/s per DOM (6 bytes/photon). The Central Logic Board (CLB, Fig. 2, E) [15] contains the hardware to control all instrumentation in the DOM and to process the produced data. It forms the interface with the fibre-optic network 
through which the data is sent and control commands are received. Each DOM is a node in an IP network and therefore has its own IP address. At the heart of the CLB is a Xilinx Kintex 7 field programmable gate array (FPGA). The FPGA contains time to digital (TDC) channels for the PMTs and the acoustic sensor and a pipeline to process all data to IP/UDP packets. The data are collected in frames of $100 \mathrm{~ms}$ length and split into UDP packets (jumbo frames) which are sent over the IP network to be assembled by dedicated computers on shore. Two LM32 processors [16] are configured on the FPGA. The first is part of a white-rabbit PTP (precision time protocol) core [17] which manages the timing [18] and communication with the outside world via IP. The second LM32 allows for direct access to the registers in the read-out pipeline, and the I2C and SPI busses and UARTS that connect to the different instruments and components. The FPGA is configured (firmware and software) from a flash memory at power-up. The firmware and hardware image can be re-programmed on the flash from the FPGA which can be accessed via the ethernet connection. A secure fall-back image is present, ensuring communication with the DOM. A small form-factor plugable (SFP) cage on the CLB is equipped with a single-mode laser transceiver at DOM production. Each DOM within a detection unit uses a unique wavelength for transmitting data. Incoming (control) data is received on a broadcast channel, common to all CLBs. An add/drop filter is used to combine the transmit and receive channels onto the one fibre passing through the penetrator.

Two signal collection boards (octopus boards) (Fig. 2, F) carry the LVDS signals from the PMTs to the CLB from where the signals are routed to the FPGA. The signal collection boards connect the PMTs to an I2C bus. One board connects to the 12 PMTs of the top hemisphere while the other connects to the 19 PMTs from the bottom hemisphere and to the acoustic sensor. Power to the systems is provided by a dedicated printed circuit board, the power board (Fig. 2, D). This board can be monitored and controlled via the FPGA. The power board is fed with $12 \mathrm{~V}$ DC which is led into the DOM via the penetrator from the DC/DC converter in the break-out-box external to the glass sphere. An attitude and heading reference system (AHRS) mezzanine board is mounted on the CLB which provides compass, tilt- and accelerometer data. This data is used to reconstruct the orientation and position of a DOM in the water, in conjunction with the acoustic positioning system. Temperature and humidity sensors are mounted both on the CLB and on the power board. Care has been taken to achieve a low power use. The total power consumption of a DOM with all instruments operating is around 7 Watt.

\section{Conclusion}

The innovative design of the KM3NeT multi-PMT Digital Optical Module allows to fully exploit the potential of Mediterranean Sea water in uncovering the sources of neutrinos and studying neutrino properties with unprecedented accuracy. A prototyping program has validated the DOM design and proven its physics potential [20] and [21]. Results from the prototypes have demonstrated the photon counting, background rejection capabilities and direction sensitivity of single multi-PMT DOMs. Novel developed algorithms which extract the properties of the interacting neutrinos make use of the characteristics of the KM3NeT DOMs. For example, an algorithm that can determine the direction of neutrino induced particle cascades [22] to better than a few degrees makes use of the photon counting capabilities and the directional sensitivity of the DOM. The 
design and prototyping phases have concluded succesfully and the mass production of KM3NeT DOMs has started.

\section{References}

[1] http://www.km3net.org

[2] P. Piattelli (KM3NeT Collaboration), All-flavour high-energy neutrino astronomy with KM3NeT/ARCA in proceedings of $34^{\text {th }}$ ICRC, The Hague, The Netherlands, PoS(ICRC2015)1158 (2015)

[3] D. Calvo (KM3NeT Collaboration), Nanobeacon: a low cost time calibration instrument for the KM3NeT neutrino telescope, VLVnT13 proceedings (2014)

[4] A. Enzenhöfer (KM3NeT Collaboration), Integration of acoustical sensors into the KM3NeT Optical Modules, AIP Conf.Proc. 1630 (2014) 189-192 (2014)

[5] S. Viola (KM3NeT Collaboration), Acoustic positioning system for KM3NeT in proceedings of $34^{\text {th }}$ ICRC, The Hague, The Netherlands, PoS(ICRC2015)1169 (2015)

[6] http://arxiv.org/abs/1304.0697

[7] R. Bormuth et al. (KM3NeT Collaboration), Characterization of the ETEL and HZC 3-inch PMTs for the KM3NeT project, AIP Conference Proceedings 1630 (2014) 114

[8] S. Aiello et al. (KM3NeT Collaboration), Characterization of the 80-mm diameter Hamamatsu PMTs for the KM3NeT project, AIP Conf. Proc. 1630 (2014)

[9] P. Timmer et al., Very low power, high voltage assembly cabase for a Photo Multiplier Tube for the KM3NeT deep sea neutrino telescope, JINST 5, C12049 (2010)

[10] D. Gajanana et al., ASIC design in the KM3NeT detector, JINST 8, C0203 (2013)

[11] D. Gajanana et al. A front end ASIC for the readout of the PMT in the KM3NeT detector, JINST 5, C12040 (2010)

[12] C. Mollo (KM3NeT Collaboration), The dark box instrument for fast automatic testing of the photomultipliers for KM3NeT in proceedings of $34^{\text {th }}$ ICRC, The Hague, The Netherlands, PoS(ICRC2015)1159 (2015)

[13] S. Adrián-Martínez et al. (KM3NeT Collaboration), Expansion cone for the 3-inch PMTs of the KM3NeT optical modules, JINST 8, T03006 (2013)

[14] S. Biagi (KM3NeT Collaboration), The data acquisition system of the KM3NeT detector in proceedings of $34^{\text {th }}$ ICRC, The Hague, The Netherlands, PoS(ICRC2015)1172 (2015)

[15] S. Biagi and A. Orzelli (KM3NeT Collaboration), The Central Logic Board and its auxiliary boards for the optical module of the KM3NeT detector, JINST 9 (2014) C12033.

[16]

http://www.latticesemi.com/en/Products/DesignSoftwareAndIP/IntellectualProperty/IPCore/IPCores02/LatticeMico32.aspx

[17] http://www.ohwr.org/projects/wr-cores/wiki/wrpc_core

[18] M. Bouwhuis (KM3NeT Collaboration), Time synchronization and time calibration in KM3NeT in proceedings of $34^{\text {th }}$ ICRC, The Hague, The Netherlands, PoS(ICRC2015)1170 (2015)

[19] P. M. Kooijman (KM3NeT Collaboration), The Mechanical structure and deployment procedure of the KM3NeT detection unit in proceedings of $34^{\text {th }}$ ICRC, The Hague, The Netherlands, PoS(ICRC2015)1173 (2015)

[20] S. Adrián-Martínez et al. (KM3NeT Collaboration), Deep sea tests of a prototype of the KM3NeT digital optical module, Eur. Phys. J. C 74 (2014) 9, 3056

[21] S. Biagi and A. Creusot (KM3NeT Collaboration), Performances and main results of the KM3NeT prototypes, in proceedings of $34^{\text {th }}$ ICRC, The Hague, The Netherlands, PoS(ICRC2015)1164 (2015)

[22] D. Stransky (KM3NeT Collaboration), Reconstruction of cascade-type neutrino events in KM3NeT/ARCA in proceedings of $34^{\text {th }}$ ICRC, The Hague, The Netherlands, PoS(ICRC2015)1108 (2015) 